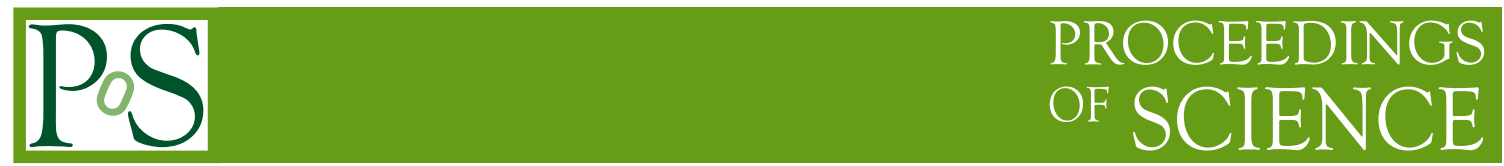

\title{
Nuclear Matrix Elements for Neutrinoless Double Beta Decay
}

\author{
Jonathan Engel* \\ University of North Carolina \\ E-mail: engelj@physics.unc.edu
}

\begin{abstract}
Nuclear-structure theory has advanced quickly in recent years. The field is now at the point at which an accurate calculation of the nuclear matrix elements governing neutrinoless double-beta decay should soon be possible. I describe some promising theoretical many-body methods and their application to double-beta decay.
\end{abstract}

38th International Conference on High Energy Physics 3-10 August 2016

Chicago, USA

${ }^{*}$ Speaker. 
Neutrinoless double-beta $(0 v \beta \beta)$ decay occurs, if the three neutrinos we know about are Majorana particles, at a rate that depends on a weighted average of neutrino masses $[1,2,3]$. New experiments to search for $0 v \beta \beta$ decay are under development, and one or more ton-scale experiments may be funded. Extracting a mass from the results, however, or setting a reliable upper limit — even planning the experiments efficiently — will require accurate values of the nuclear matrix elements governing the decay. These cannot be measured and so must be calculated.

The matrix elements have been computed in traditional nuclear models, but vary by factors of two or three. Nuclear-structure theory is now at the point, however, where so-called ab initio (or "from first-principles") calculations in systems with up to 50 or 60 nucleons are possible, and those in heavier nuclei should soon be possible as well. One starts with an interaction among nucleons, usually obtained from chiral effective field theory, and solves the many-nucleon Schrödinger equation in a controlled approximation scheme with quantifiable errors. In this paper, I review some of the more promising $a b$ initio schemes for computing the double-beta matrix elements. I also briefly discuss the currently unsettling "renormalization" of the nuclear weak axial coupling constant $g_{A}$, and argue that the cause will be identified soon.

The lifetime for neutrinoless double-beta decay, if the exchange of the familiar light neutrinos is responsible, is given by the product of a phase space factor, an average neutrino mass, and a nuclear matrix element $M^{0 v}$. The matrix in the widely used (and reasonably accurate) "closure approximation" and without two-nucleon weak currents (which I take up briefly later) is

$$
\begin{aligned}
& M^{0 v}=\frac{2 R}{\pi g_{A}^{2}} \int_{0}^{\infty} q d q \\
& \quad \times\left\langle f\left|\sum_{a, b} \frac{j_{0}\left(q r_{a b}\right)\left[h_{F}(q)+h_{G T}(q) \vec{\sigma}_{a} \cdot \vec{\sigma}_{b}\right]+3 j_{2}\left(q r_{a b}\right) h_{T}(q) \vec{\sigma}_{a} \cdot \vec{r}_{a b} \vec{\sigma}_{b} \cdot \vec{r}_{a b}}{q+\bar{E}-\left(E_{i}+E_{f}\right) / 2} \tau_{a}^{+} \tau_{b}^{+}\right| i\right\rangle,
\end{aligned}
$$

where $r_{a b} \equiv\left|\vec{r}_{a}-\vec{r}_{b}\right|$ is the distance between nucleons $a$ and $b, j_{0}$ and $j_{2}$ are the usual spherical Bessel functions, $\bar{E}$ is an average excitation energy to which the matrix element is insensitive, and the nuclear radius $R \equiv 1.2 A^{1 / 3} \mathrm{fm}$ is inserted to make the matrix element dimensionless. The function $h_{F}, h_{G T}$, and $h_{T}$ are Fermi, Gamow-Teller, and tensor "form factors," defined in Ref. [4]. Similar matrix elements would govern the exchange of heavy particles, e.g. right-handed neutrinos or supersymmetric particles, that might also mediate the decay.

$A b$ initio approaches usually employ chiral effective field theory to determine the interactions among nucleons. The theory [5] is designed for energies of up to a few hundred $\mathrm{MeV}$, so the only degrees of freedom are nucleons and pions. The chiral framework, which provides a counting scheme in powers of momentum or pion mass divided by a QCD scale $\Lambda_{Q C D}$ of about $500 \mathrm{MeV}$, allows one to write down all possible interactions that are consistent with the underlying spontaneously broken chiral symmetry at any order of $p / \Lambda_{Q C D}$ or $m_{\pi} / \Lambda_{Q C D}$. Two-nucleon interactions begin with one Feynman digram at leading order, and three-nucleon interactions with two diagrams at next-to-next-to leading order. The coefficients of the interactions up to any order are fit to data. As long as one truncates the theory at some order $n$, one has a Hamiltonian that should reproduce any low-energy data up to $\mathscr{O}\left(\left[p / \Lambda_{Q C D}\right]^{n+1}\right)$. These Hamiltonians now typically include terms in next-to-next-to-next-to leading order $\left(\mathrm{N}^{3} \mathrm{LO}\right)$ in the chiral expansion.

Several many-body methods can be used in conjunction with the chiral Hamiltonians. Some nuclei are easier to apply such methods to than others. The easiest are those that can be represented 


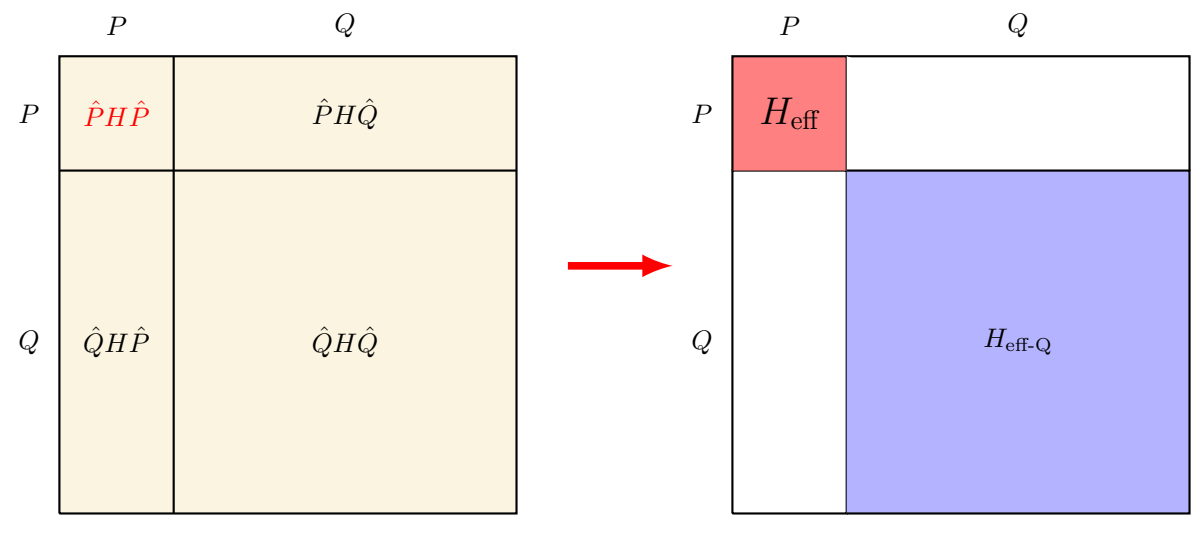

Figure 1: Representation of unitary transformation for constructing effective shell-model Hamiltonian $H_{\text {eff }}$. The operator $\hat{P}$ projects onto the shell-model (valence) space with an inert core, and the operator $\hat{Q}$ projects onto onto the rest of the many-body Hilbert space. After the transformation, the two spaces are decoupled.

in first approximation by a single spherical Slater determinant. Such nuclei have "closed shells," i.e. a specific number of protons or neutrons so that the nucleus is much more bound than the nucleus with one additional proton or neutron. Many-body methods handle these nuclei by systematically correcting the Slater determinant. "Open-shell" nuclei are more difficult because they require a more sophisticated starting point. But they are the ones relevant to double-beta decay.

Open-shell nuclei have been described for a long time by what is called the nuclear shell model. The model focuses on the nucleons outside the last closed shell(s), with the others taken to form an inert core. The last (valence) nucleons are allowed to correlate arbitrarily, though only within a few valence single-particle orbitals. For that reason the Hamiltonian described in the previous section must be replaced by an effective Hamiltonian. Once that Hamiltonian has been determined, traditionally through fits to nuclear data in isotopes close to those one cares about, the model works quite well. In our context, however, it has a problem. We are computing the matrix element of a complicated two-body nuclear operator. The "bare operator" of Eq. (1) may not be sufficient in a limited space, for the same reason the bare Hamiltonian is not. Thus, even if the model describes spectra well, it may err considerably in its predictions of double-beta matrix elements.

Researchers are now, however, beginning to map ab inito calculations into shell-model calculations. Among the ab initio approaches in which this can be done are coupled clusters theory [6] and the in-medium similarity renormalization group (IM-SRG) [7]. The idea behind both approaches is to construct a unitary transformation that puts the full many-body Hamiltonian in block diagonal form, with a piece $H_{\text {eff }}$ in the shell-model space that reproduces the lowest-lying energies exactly. Figure 1 presents the idea graphically, in terms of projection operators typically labeled $\hat{P}$ and $\hat{Q}$.

Coupled cluster theory is based on replacing a single Slater determinant with the completely general correlated state vector:

$$
\begin{aligned}
\mid \text { corr }\rangle & =e^{T}|S D\rangle \\
T & =\sum_{m i} t_{m i} a_{m}^{\dagger} a_{i}+\sum_{m n i j} t_{m n, i j} a_{m}^{\dagger} a_{n}^{\dagger} a_{i} a_{j}+\ldots
\end{aligned}
$$

Here $|S D\rangle$ is the Slater determinant and $i, j$ label occupied orbitals in that determinant while $m, n$ 
label empty orbitals. Thus the operator $T$ creates one-particle one-hole configurations, two-particle two-hole configurations, etc. The presence of these operators in the exponent means the correlations are iterated and many-particle many-hole excitations are present, even when the series is truncated at the point indicated in Eq. (2).

Coupled clusters theory has reached the point at which the correlated closed-shell state above can be generalized to states containing two or three valence particles outside closed shells. The procedure for obtaining a shell-model interaction, e.g. in a valence space appropriate for ${ }^{76} \mathrm{Ge}$ (a prime double-beta decay candidate) goes something like this:

1. Begin with a two- and three-nucleon Hamiltonian from chiral effective field theory [8].

2. Do ab initio coupled-clusters calculations of the ground state of the closed shell nucleus ${ }^{56} \mathrm{Ni}$, of the low-lying eigenstates states of the closed-shell-plus-one nuclei ${ }^{57} \mathrm{Ni}$ and ${ }^{57} \mathrm{Cu}$, and of the low-lying states of the closed-shell-plus-two nuclei ${ }^{58} \mathrm{Ni},{ }^{58} \mathrm{Cu}$, and ${ }^{58} \mathrm{Zn}$. Eventually, when it becomes possible, do the same in closed-shell+three nuclei as well.

3. Perform a "Lee-Suzuki" mapping [9] of the low-lying states in these nuclei onto states in the valence shell containing one and two (and eventually, three) nucleons. The mapping is designed to maximize the overlap of the full ab initio eigenstates with their shell-model images, while preserving orthogonality of the images.

4. Use the mapping of states to construct the shell-model interaction $H_{\text {eff }}$ that gives the image states the same energies as their parents. Construct an effective double-beta operator that gives the same matrix elements between image states as the bare operator does between the associated parents. The formalism that allows this step was worked out long ago.

5. Put 4 protons and 16 neutrons (for ${ }^{76} \mathrm{Ge}$ ) and 6 protons and 14 neutrons (for ${ }^{76} \mathrm{Se}$ ) in the valence shell and use the effective interaction and decay operator derived in the previous step to calculate the ground-state-to-ground-state decay matrix element.

The last step, of course, is where things get tricky, because in principle, many-body effective interactions will be needed once more nucleons are in the valence shell. There are arguments that the effects of these fall off with the number of nucleons involved in the interaction. There is no power-counting scheme that tells us how fast, but one expects three-body interactions and operators to be sufficient. The expectation can be checked by including four-body operator approximately. Such checks are how error is estimated in ab initio work.

The program is just now getting underway, with the first calculations in relatively light nuclei. Reference [6] uses coupled cluster calculations in ${ }^{16,17,18} \mathrm{O}$ to predict the spectra of oxygen isotopes with more neutrons. Figure 2 shows the results for ${ }^{22} \mathrm{O}$ and ${ }^{23} \mathrm{O}$ alongside those of the IM-SRG, to be discussed shortly. The left column for each isotope contains the "coupled-cluster effectiveinteraction" (CCEI) predictions, the right column the experimental data, and the second-to-right column the "predictions" of the USD shell-model interaction [10] that was fit long ago to lots of data in neighboring nuclei. The coupled-clusters-generated interaction, which uses only data in two- and three-nucleon systems to obtain the $\mathrm{N}^{3} \mathrm{LO}$ chiral interaction, produces results that are just as good. Though researchers have yet to investigate matrix elements of the double-beta operator, the initial results for energy levels are extremely promising. 

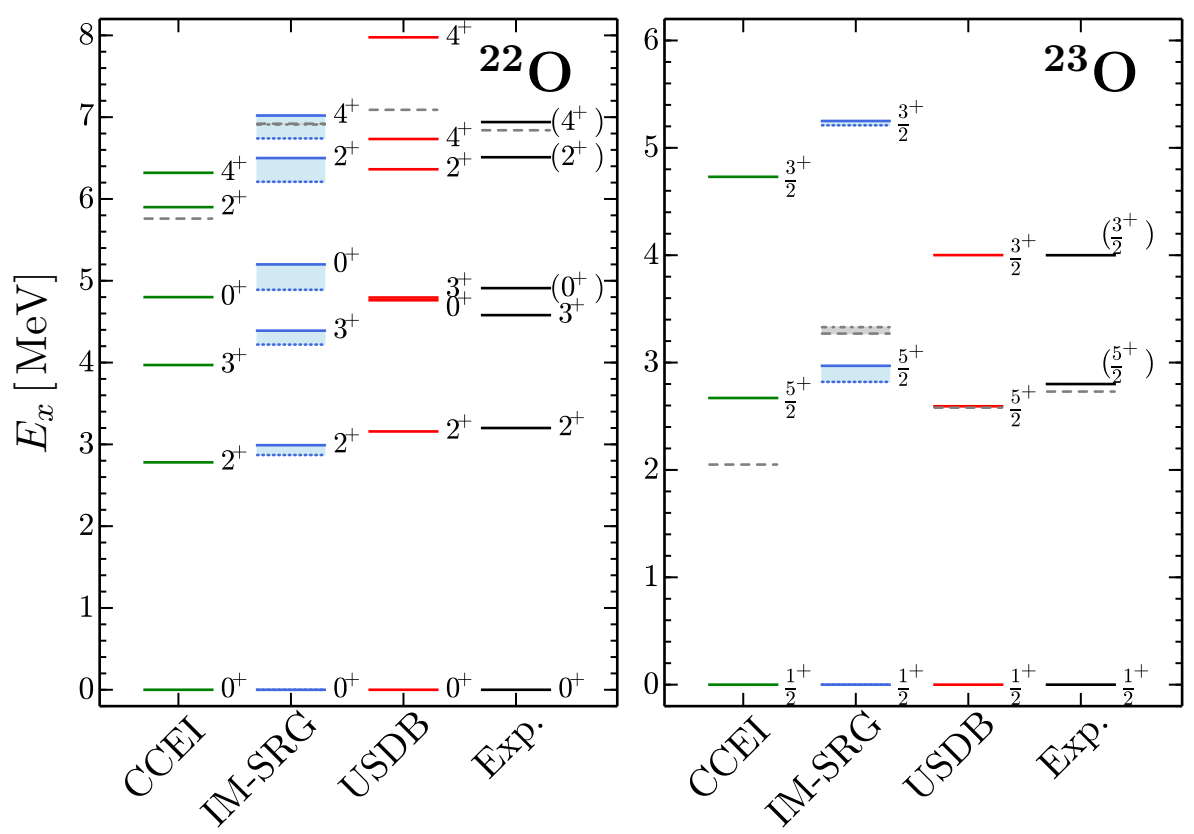

Figure 2: Spectra of two neutron-rich oxygen isotopes. The left column contains the results of $s d$-shellmodel calculations with an effective Hamiltonian derived form ab initio chiral two- and three-body forces [8] and coupled-cluster calculations in ${ }^{16,17,18} \mathrm{O}$. The next column contains the predictions of the IM-SRG. The third column contains predictions with the phenomenological USD interaction [10] that was fit to data in the same shell, and the right column contains experimental data. Figure taken from Ref. [11].

A similar program is being undertaken in the IM-SRG; it has actually been taken a bit further and is a bit more flexible [7]. The idea of that method is to write down flow equations for the unitary transformation that decouples the shell-model space from everything else, that is, to decouple the spaces incrementally. The flow equations are subject to truncation, like the operator in Eq. (2), but they are truncated at each step in the flow. The method takes advantage of normal ordering with respect to the decoupled space to minimize the effects of truncation. Figure 2 shows that the approach works as well as the coupled-clusters methods in the oxygen isotopes.

I turn finally to the renormalization of the axial-vector coupling $g_{A}$. It has been known for some time [10] that matrix elements for single-beta and two-neutrino double-beta decay are smaller in reality than in our calculations. If $0 v \beta \beta$ matrix elements are as small compared to our calculations as two-neutrino matrix elements, experiments will be less capable then we have thought. Fortunately, the issue can now be investigated systematically. There can only be two sources of the quenching: many-body weak currents, which would alter the expression in Eq. (1), and model space truncation, i.e. the omission of important configurations. Work is now beginning to examine both these sources. The effects of many-body currents have traditionally been thought to be small, but the construction of those currents in chiral effective field theory - currents that should go along with the interactions used by ab initio calculations - may lead to larger effects [12, 13]. Crucially, however, those effects should be smaller for neutrinoless decay than for two-neutrino decay. Careful EFT parameter fits should allow us to understand the effects of many-body currents on the various kinds of decay in the near future. The other source of quenching, model-space truncation, can 
be investigated in the ab initio shell-model calculations described above. Those implicitly include many configurations from outside shell-model spaces in the effective interactions and operators. We should soon be able to see whether neutrinoless decay is quenched, and if so, by how much.

\section{Acknowledgments}

I am grateful to collaborators G. Hagen, G.R. Jansen, J. Menéndez, A. Signoracci, F. Šimkovic, and P. Vogel. Support for this work was provided through the Scientific Discovery through Advanced Computing (SciDAC) program funded by US Department of Energy, Office of Science, Advanced Scientific Computing Research and Nuclear Physics, under Contract No. DE-SC0008641, ER41896, and by the U.S. Department of Energy under Contract No. DE-FG02-97ER41019.

\section{References}

[1] F. T. Avignone III, S. R. Elliott, and J. Engel, Double Beta Decay, Majorana Neutrinos, and Neutrino Mass, Rev. Mod. Phys. 80 (2008) 481.

[2] J. D. Vergados, H. Ejiri, and F. Šimkovic, Theory of neutrinoless double beta decay, Rep. Prog. Phys. 75 (2012) 106301.

[3] Jonathan Engel and Javier Menéndenz, Status and Future of Nuclear Matrix elements for neutrinoless double-beta Decay: a review, arXiv:1610.06548 (2016).

[4] F. Šimkovic, G. Pantis, J. D. Vergados, and A. Faessler, Additional nucleon current contributions to neutrinoless double $\beta$ decay, Phys. Rev. C 60 (1991) 055502.

[5] See, e.g., Evgeney Epelbaum, Hans-Werner Hammer, and U-G. Meissner, Modern theory of nuclear forces, Rev. Mod. Phys. 81 (2009) 1773.

[6] G. Jansen, J. Engel, G. Hagen, P. Navratil, and A. Signoracci, Ab-Initio Coupled-cluster effective interactions for the shell model: application to neutron-rich oxygen and carbon isotopes, Phys. Rev. Lett. 113 (2014) 142502.

[7] S. R Stroberg, H. Hergert, J. D. Holt, S. K. Bogner, and A. Schwenk, Ground and excited states of doubly open-shell nuclei from ab initio valence-space Hamiltonians, . Rev. C 93 (2016) 051301.

[8] D. R. Entem, and R. Machleidt, Accurate charge-dependent nucleon-nucleon potential at fourth order of chiral perturbation theory, Phys. Rev. C 68 (2003) 041001.

[9] Ak. F. Lisetskiy, M. K. G. Kruse, B. R. Barrett, P. Navratil, I. Stetcu, and J. P. Vary, Effective operators from exact many-body renormalization, Phys. Rev. C 80 (2009) 024315.

[10] B. A. Brown, and B. H. Wildenthal, Status of the nuclear shell model, Annu. Rev. Nucl. Part. Sci. 38 (1988) 29.

[11] H. Hergert, S. K. Bogner, T. D. Morris, A. Schwenk, and K. Tsukiyama, The in-medium similarity renormalization group: a novel ab initio method for nuclei, Phys. Rep. 621 (2016) 165.

[12] J. Menéndez, D. Gazit, and A. Schwenk, chiral two-body currents in nuclei: Gamow-Teller transitions and neutrinoless double-beta decay, Phys. Rev. Lett. 107 (2011) 062501.

[13] J. Engel, F. Šimkovic, and P. Vogel, Chiral Two-body currents and neutrinoless double-beta decay in the QRPA, Phys. Rev. C 89 (2014) 064308. 